\title{
REVISIONES
}

\section{Dimensiones, características e indicadores para una escuela democrática****}

\author{
Dimensions, characteristics and indicators for a democratic school
}

\author{
Jordi Feu Gelis, ${ }^{a}$ Núria Simó Gil, ${ }^{b}$ Carles Serra Salamé, ${ }^{a c}$ \\ Joan Canimas Bruguéad \\ ${ }^{a}$ Departamento de Pedagogía. Universidad de Girona. Cataluña \\ Telf.: (34) 9724180 12. Correo electrónico: jordi.feu@udg.edu \\ ${ }^{\mathrm{b}}$ Departamento de Pedagogía. Universidad de Vic-UCC. Cataluña \\ Telf.: (34) 9388161 64. Correo electrónico: nuria.simo@uvic.cat \\ cTelf.: (34) 249724183 00. Correo electrónico: carles.serra@udg.edu \\ dTelf.: (34) 9724189 87. Correo electrónico: joan.canimas@udg.edu
}

\section{RESUMEN}

El objetivo principal del artículo es presentar una conceptualización teórica de democracia acompañada de una operativización basada en las principales dimensiones que, a nuestro entender, definen el término. Dicho artículo parte del análisis conceptual desarrollado por el equipo Demoskole en el marco de la investigación "Democracia y participación y educación inclusiva en los centros educativos". El artículo se divide en tres secciones principales: en la primera definimos de manera clara qué entendemos por democracia y democracia en la escuela, en la segunda presentamos los ítems de las cuatro dimensiones básicas establecidas por el equipo (gobernanza, habitanza, alteridad y valores, virtudes y capacidades) que hemos definido en el apartado anterior y en la última sección presentamos las conclusiones y algunos aspectos de discusión acordes con aspectos teóricos y prácticos que forman parte del debate actual.

Palabras clave: democracia, democracia y escuela, democratización escolar, participación educativa, ciudadanía.

\begin{abstract}
The purpose of this article is to portray a theoretical conceptualization of democracy along with an operativization based upon the main dimensions which, according to our framework, define the concept. The article starts with the conceptual analysis developed by the Demoskole team in the context of the research "Democracy and participation and inclusive education in educational institutions". This job is divided into three main areas: first, a
\end{abstract}

Demoskole: Democracia, participación y educación inclusiva en los centros educativos es una Investigación I+D de tres años del Plan Nacional financiada por el Ministerio de Educación y Competitividad (MINECO, 2013-2015). Coordinadores: Jordi Feu y Núria Simó.

** Si bien la autoría de este artículo pertenece a las personas mencionadas, buena parte de las reflexiones que aquí aparecen han sido formuladas por los investigadores que integran el grupo Demoskole. Por parte de la Universidad de Girona: Paco Abril, Xavier Besalú, Joan Canimas, M. Falgàs, Laura Lázaro, Patricia Melgar, Eduard Mondéjar, Gloria Muñoz, Josep M. Palaudarias, Òscar Prieto y Carles Serra, y por parte de la Universidad Vic: Mar Beneyto, Isabel Carrillo, Laura Domingo, Laura Farré, Esther Fatsini, Cati Lecumberri, Alba Parareda, Joan Soler, Itxaso Tellado y Antoni Tort. La organización de los datos recopilados para dicho artículo ha contado con la colaboración de una de las investigadoras en formación de proyecto: Laura Lázaro. 
clear definition of what we understand by democracy and school democracy; second, a presentation of the items of the four basic dimensions established by the team (governance, inhabitancy, otherness and values, virtues and abilities) defined in the previous section; and finally, the conclusions and some discussion related to theoretical and practical aspects which are present in the current debate.

Key words: democracy, democracy and school, school democratization, educational participation, citizenship.

\section{INTRODUCCIÓN}

Al pisar la arena de los centros educativos españoles, se tiene a menudo la impresión de que la idea de lo que significa democracia es más bien simple, excesivamente complaciente y escasamente comprometedora. En términos generales, se diría que se trata de un concepto blando, impreciso, incluso pobre. Lo más frecuente es que se haga referencia a ella a partir del relato de experiencias concretas, más o menos relacionadas con dos valores recurrentes (la participación y la igualdad) y que se omita toda elaboración teórica que ayude a precisar de qué se habla exactamente, por lo que es una visión un tanto limitada de la vida y la calidad democrática de los centros educativos.

Nos atreveríamos a afirmar que en la escuela sucede algo parecido a lo que Wendy Brown observó para el conjunto de la sociedad: en ella, dijo, "la democracia goza de una popularidad sin precedentes, [...] pero lo cierto es que, en términos conceptuales, nunca ha adolecido de un carácter más indefinido y sustancialmente vacío" (Brown, 2010, p. 59). A partir del trabajo de campo que el grupo Demoskole ha realizado hasta el día de hoy, hemos llegado a la conclusión de que una parte importante de docentes, alumnos y familias tienen serias dificultades para explicar qué entienden por democracia, y todavía les resulta más difícil explicar qué entienden por escuela democrática.

Asimismo, da la impresión de que la cultura escolar dominante equipara la democracia con la participación en órganos estandarizados ${ }^{1} \sin$ apenas cuestionarse algunos aspectos clave de la calidad democrática. La Ley Orgánica de Mejora de la Calidad del Sistema Educativo (LOMCE, 8/2013, de 9 de diciembre) produjo cierto revuelo en torno a las competencias que los consejos escolares tienen, pero dicho revuelo puso en evidencia el desconocimiento que un gran número de docentes, buena parte de los grupos de presión y de los académicos, tienen de la democracia vivida en los centros educativos y de la función que históricamente han tenido dichos consejos, que nunca ha sido relevante, si por ello entendemos la capacidad de hacer política al servicio de la comunidad educativa. ${ }^{2}$

$\mathrm{Si}$ bien algunas investigaciones realizan valoraciones positivas de la calidad democrática de nuestros centros educativos (González, 2004), prevalecen aquellas que son críticas con esta cuestión (Feito, 2010; Fernández Enguita, 1992; Garreta, 2009; Gil Villa, 1995; Santos Guerra, 1999). La baja participación del alumnado y de las familias, la ausencia de verdaderos procesos deliberativos, el escaso debate sobre cuestiones centrales en la vida de los centros, la burocratización de la gestión, etc., son elementos clave en la desnaturalización del espíritu originario de los consejos. Una desnaturalización que preocupa al mismo Consejo Escolar del Estado (basta con leer algunos de los informes

Fundamentalmente, consejo escolar de centro, claustro de profesores, junta de la asociación de padres y madres y junta de delegados.

Para más información, sugerimos la lectura del artículo de Feito (2014). 
o dictámenes que emite dicha institución, incluso cuando es presidida por sectores conservadores) $)^{3}$.

En las investigaciones previas del grupo Demoskole, los análisis de los órganos de participación colegiados e institucionalizados pusieron en duda algunos aspectos del modelo participativo, tales como la representatividad de sus integrantes, la legitimad de los acuerdos adoptados, la capacidad de sus miembros para trasladar la información al resto del grupo, etc.

Así pues, tras la revisión de la literatura científica hasta aquí referenciada y de las investigaciones del grupo Demoskole podemos afirmar que:

a. La mayoría de los profesionales de los centros educativos y de los trabajos desarrollados en el ámbito académico relacionan la democracia casi exclusivamente con el desarrollo de las estructuras y prácticas de gobernanza. Frente a ello, consideramos que es preciso introducir en el debate profesional y académico otras dimensiones de la democracia que nos permitan ir más allá del formalismo democrático, de la implementación de estructuras de gobierno, del impulso de órganos representativos y del fomento de espacios de participación. Como defenderemos en este artículo, el grupo Demoskole recupera la idea de Dewey (1895, p. 224) de la escuela como "una institución que sea, provisionalmente, un lugar de vida para el niño, en la que este sea un miembro de la sociedad, tenga conciencia de su pertenencia y a la que contribuya" para fundamentar que la democracia no se agota en las estructuras o las prácticas de gobernanza: es también una forma de ser y estar en sociedad, una forma de relacionarse con los demás y unos valores sin los cuales estas dimensiones no son posibles.

b. Las formas de gobernanza identificadas en la mayoría de los centros son más bien convencionales, con tendencia a reproducir el sistema participativo de las instituciones políticas del Estado y apenas contribuyen a la democratización real de la vida de los centros y a la extensión de las prácticas y valores democráticos al conjunto de la sociedad. A pesar de que, tanto por masa crítica de la mayoría de centros como por oportunidad y conveniencia educativa se hubiera podido optar por un modelo más participativo y extensivo, próximo a los principios de la democracia republicana, deliberativa y participativa (más cercanos a los discursos y propuestas actuales relacionadas con la renovación y profundización democrática).

\section{METODOLOGÍA}

La metodología para la obtención de los resultados que aquí se presentan ha sido la siguiente: (i) estudio y análisis de la bibliografía más relevante sobre el tema; (ii) debate, identificación y definición de las cuatro dimensiones de la democracia que se proponen (gobernanza, habitanza, alteridad y éthos) y de los indicadores de cada una de ellas; (iii) trabajo de campo con una metodología de investigación participativa en diez centros educativos; (iv) análisis e incorporación de los resultados del trabajo de campo; y $(v)$ discusión y conclusiones.

En el trabajo de campo, realizado en diez centros educativos, se ha optado por la metodología de investigación participativa (Kesby, Kindon y Pain, 2007): los resultados del grupo investigador (las cuatro dimensiones de la democracia y la primera propuesta de

Para más información: Consejo Escolar del Estado, Informe sobre el estado y situación del sistema educativo. Curso 2007 2008, p. 24; y si se quiere un informe más actualizado, véase Informe 2013 sobre el estado del sistema educativo. Curso 2011-2012, p. 602-604 (sección «Propuestas de mejora»). 
indicadores de cada una de ellas) se han contrastado con profesorado, alumnado y familias de los diez centros educativos; y los resultados de estos grupos de discusión se han ido incorporando a la investigación con el fin de desarrollar el modelo teórico.

Los criterios de selección de los centros educativos han sido: (i) distintas etapas educativas (cinco centros de infantil, primaria y cinco de secundaria); (ii) centros educativos con prácticas democráticas reconocidas; (iii) distintos entornos geográficos (ocho centros educativos pertenecientes a un medio urbano y dos a un rural); ( $i v)$ titularidad (ocho centros públicos, uno concertado y uno privado); y (v) la voluntad explícita de cada centro de participar en la investigación.

\section{3. ¿QUÉ ENTENDEMOS POR DEMOCRACIA Y POR DEMOCRACIA EN LA ESCUELA?}

\subsection{EL CONCEPTO DE DEMOCRACIA}

Antes de establecer las dimensiones e indicadores de lo que consideramos que son escuelas democráticas, vamos a definir qué entendemos por democracia. Nuestra voluntad es elaborar un concepto comprehensivo, coherente y delimitado, y que al mismo tiempo sea posible objetivarlo y pueda implementarse en cualquier centro escolar.

El concepto de democracia por el que apostamos integra los postulados de las tres generaciones de derechos humanos y sociales, y se alinea con los principios de la democracia republicana, deliberativa y participativa. Partimos, pues, de las aportaciones de autores como Taylor (1979), Fishkin (1995, 2009), Habermas (1999), Sandel (1998), De Francisco (1998), Pettit (1998), Elster (2001), Viroli (2002), Barber (2004), Skinner (2004), Martí (2006) y Subirats (2011), quienes de una manera u otra ejercen una crítica al modelo de democracia liberal representativa, por considerarla elitista, competitiva y rígida en la distinción que establece entre gobernantes y gobernados, y por considerar que la ciudadanía no está suficientemente representada.

Por otra parte, el modelo que proponemos se estructura en base al progresivo reconocimiento de derechos desarrollado por las denominadas tres generaciones de derechos humanos. Como es sabido, los derechos humanos de primera generación fueron formulados a finales del siglo XVIII, en la Declaración de los Derechos del Hombre y del Ciudadano de 1789 y en la Carta de Derechos de los Estados Unidos de 1791, y tratan principalmente de la libertad y la participación en la vida política y de las formas y límites en la gestión del poder. Sin embargo, con el tiempo se hizo evidente que estas garantías no eran suficientes y que se requerían unas condiciones de vida, unos derechos económicos, sociales y culturales que se conocen como de segunda generación y que, junto con la primera, quedaron plasmados en la Declaración Universal de Derechos Humanos de 1948. Asimismo, a partir de los años ochenta del siglo XX se hizo a su vez evidente que también era necesario el reconocimiento y la fraternidad con los otros y el respeto por el planeta, y se empezaron a formular derechos como el de autodeterminación, diferencia, paz, medio ambiente preservado, sano y sostenible, etc. Estos derechos, llamados de tercera generación, tratan esencialmente del reconocimiento del otro y de lo otro.

Aun a riesgo de simplificar, consideramos que los derechos humanos de la primera generación atienden principalmente a cuestiones de gobernanza, los de segunda generación, 
a cuestiones de lo que el grupo Demoskole ha denominado habitanza, y los de tercera generación a cuestiones relacionadas con la alteridad. De ahí que el concepto de democracia que proponemos se fundamente en estas tres dimensiones, más una cuarta dimensión, transversal a todas las anteriores, que denominamos éthos y que se refiere a los valores, virtudes y capacidades que debe promover toda sociedad democrática. En Feu et al. (2013) desarrollamos estas cuatro dimensiones y las justificamos, mostrando de qué manera se vinculan a los discursos actuales de la ciencia política, la filosofía y la sociología.

\subsection{ESCUELA DEMOCRÁTICA}

La democracia es una forma de gobernar, pero también un conjunto de prácticas y dinámicas que afectan a la comunidad. En el ámbito educativo, es el ejercicio de una práctica que parte de valores y presupuestos éticos que orientan un marco de acciones e interrelaciones. Una forma de ser y de estar, de relacionarse con los demás y de preocuparse por lo común (no exclusivamente de gestionarlo). Una forma de saber vivir, convivir, acoger y respetar tanto a los cercanos como a los que sentimos como diferentes o extraños. Para que la democracia sea efectiva, es necesario tener en cuenta, también en la escuela, las condiciones de gobernanza, de habitanza y de alteridad, así como los valores, principios y capacidades que difunden e inspiran las prácticas democráticas y de participación (Feu et al., 2013).

Otros autores ya han trabajado en esta línea ${ }^{4}$. Así, por ejemplo, Feito y López Ruiz (2008), para quienes una escuela democrática tiene una base ética que se concreta, por lo menos, en la justicia (lucha a favor de la igualdad y la equidad), la crítica (se imparte conocimiento y se denuncian las estructuras y dinámicas que impiden la justicia y generan discriminación y desigualdad) y la profesionalidad (existe un firme compromiso con el ejercicio de la profesión y esta está al servicio de los estudiantes). Algunos autores, como Leithwood, Begley y Cousin (1990), añaden que una escuela democrática es aquella que favorece la felicidad y el bienestar de todos y todas, en particular del alumnado. Esta forma de aproximarse a la democracia nos resulta especialmente atractiva, porque nos permite abordar el concepto desde una perspectiva amplia y compleja, en la que se entrelazan competencias susceptibles de ser tratadas por diferentes áreas de conocimiento, en distintos espacios y momentos.

Parafraseando a Freinet, la mejor democracia que se aprende en la escuela es la que se vive en ella. La democracia se aprende practicándola, ejercitándola y poniendo a prueba los límites y dificultades de la misma. Se aprende a través del currículum explícito y oculto. Edelstein (2011) nos propone tres vías para el aprendizaje de la democracia en la escuela: la primera consiste en fomentar el aprendizaje sobre la democracia, la segunda en aprender mediante la participación en democracia y la tercera en aprender para la democracia. Edelstein afirma que estas tres vías de aprendizaje son necesarias para la adquisición de competencias sociales que fomenten una educación democrática, un planteamiento que supera la idea de que la democracia escolar se restringe a las decisiones que toman los órganos de participación y de gestión del centro. El aprendizaje de la democracia en la

Desarrollamos el concepto a partir de las aportaciones realizadas por diferentes autores tanto en el ámbito español (Feito, 2010; Feito y López Ruiz, 2009; Martínez Bonafé, Molina y Montaner, 2003) como en el internacional (Apple, 2011; Apple y Beane, 2007; Biesta, 2009; Edelstein, 2011, entre otros). 
escuela implica la adquisición de un compromiso y responsabilidad del alumnado y el profesorado con el entorno social y la comunidad, y este objetivo solo es posible si las escuelas trabajan para transformar la participación de una organización escolar formal a la implicación activa con la comunidad.

Por su parte, Barbosa (2000) propone tres modelos para la ciudadanía democrática: (i) el modelo basado en la transmisión de contenidos y valores en torno a la democracia, principalmente relacionados con la ciudadanía; (ii) el modelo basado en la formación de hábitos democráticos en dos ámbitos: el de la toma de decisiones (gestión del centro) y el de la participación en los procesos de enseñanza y aprendizaje en los que el alumnado es protagonista. En este modelo es necesario construir un hábitat democrático formado por actitudes de solidaridad, de comprensión, de diálogo y de reconocimiento mutuo; (iii) el modelo basado en la introducción de las problemáticas actuales en las prácticas de la ciudadanía. Este modelo promueve la confrontación directa con la realidad sociopolítica fuera de la escuela. Avanzar en el desarrollo de estos tres modelos supone dinamizar prácticas democráticas con un alto grado de complejidad para la educación de la ciudadanía democrática.

Edelstein (2011), Barbosa (2000) Apple y Beane (1999), entre otros, han descrito, analizado y formulado prácticas educativas democráticas ${ }^{5}$, que consideramos que merece la pena explorar y reflexionar en torno a las condiciones de posibilidad, trascenderlas y enriquecerlas para dar respuesta al contexto socioeconómico y político actual.

\section{DIMENSIONES E INDICADORES PRESENTES EN LAS PRÁCTICAS EDUCATIVAS DEMOCRÁTICAS}

En los siguientes apartados se realiza una definición sintética de cada una de las cuatro dimensiones de la democracia (gobernanza, habitanza, alteridad y éthos) y se concretan los indicadores presentes en prácticas educativas democráticas con el fin de realizar posibles propuestas para evaluar la calidad democrática de los centros educativos.

\subsection{GOBERNANZA}

\subsubsection{Definición}

La gobernanza se refiere a las estructuras y a los procedimientos a través de los cuales se toman las decisiones políticas y se gestiona lo público. Se refiere al método o conjunto de reglas establecido para la constitución del gobierno y para la formación de las decisiones políticas, especialmente aquellas que son vinculantes para toda la sociedad (Bobbio, Matteuci y Pasquino, 1991, p. 449). De forma genérica, es una forma de convivencia social en la que sus miembros (la comunidad educativa, en nuestro caso) son libres e iguales y

\footnotetext{
Edelstein (2011) propone tres tipos de prácticas asociadas a las tres vías mencionadas anteriormente (a) autogobierno democrático en el aula como asambleas; (b) proyectos sociales; y (c) APS. Barbosa (2000) sugiere: a) desarrollar los contenidos en la asignatura educación para la ciudadanía o de forma transversal en todas las materias, b)democratizar la gestión colectiva del centro y organizar prácticas educativas centradas en el alumnado y c) Abrir la escuela al entorno desde la perspectiva de un currículum para la ciudadanía. Apple y Beane (1999) pretenden: a) organizar estructuras y procesos participativos para vivir la democracia en la escuela y b) plantear un currículum democrático. Dichas prácticas se encuentran más detalladas en los artículos respectivos, citados en la bibliografía.
} 
las relaciones sociales se establecen de acuerdo a mecanismos contractuales. De modo más particular, es una forma de organización en la que las decisiones colectivas son adoptadas por los agentes que integran la comunidad educativa mediante diferentes mecanismos de participación que garantizan la voz, opinión e intereses de todos los sectores. En definitiva, la gobernanza escolar gestiona las relaciones de poder que se establecen y vehiculan entre los agentes educativos del centro.

Utilizamos, pues, el concepto gobernanza en su sentido más genérico de formas de gobierno, procesos, dispositivos o mecanismos diseñados para la toma de decisiones. No lo utilizamos aquí en el sentido que se ha ido definiendo a partir de los años 90 como una nueva forma de gobernar caracterizada por la interacción de las instituciones de distinto nivel y por la interacción y el trabajo en red de las administraciones públicas con la sociedad civil o las organizaciones privadas (Rhodes, 1997; Subirats, 2010) ${ }^{6}$.

\subsubsection{Indicadores de una gobernanza escolar democrática}

\subsubsection{Existencia de mecanismos de participación en la toma de decisiones}

Se observará el establecimiento de procesos y mecanismos de participación en la toma de decisiones. Se considerará que dichos mecanismos propician una mayor calidad democrática si:

a. Existen encuentros o espacios de participación con una función, estructura, composición y dinámica de funcionamiento asignada.

b. Estos encuentros o espacios son revisados y sometidos a deliberación por los miembros de la comunidad educativa, con las limitaciones que establezca el marco legal (que se puede desarrollar e interpretar de modo que favorezca al máximo la participación de todos).

c. Se apuesta por formas de gobierno horizontal, exclusivamente limitadas por lo que establece el marco legal. Dicho marco legal se desarrolla e interpreta de modo que permita abrir la estructura, composición y funcionamiento de los órganos de gobierno, y fomentar al máximo la participación de los miembros de la comunidad educativa.

d. Se apuesta por formas de gobierno multinivel, en que los distintos niveles institucionalizados y no institucionalizados comparten (en lugar de monopolizar) decisiones sobre amplias áreas, y se establecen formas de legitimidad compartida.

e. Existen mecanismos para encajar las propuestas y formas de participación de carácter más espontáneo e informal que se generan en la vida social de los centros. Dichas formas de participación comparten legitimidad con las estructuras más formales.

\subsubsection{Extensión de los miembros de la comunidad educativa llamados a participar}

a. Todos los miembros de la comunidad educativa (equipo directivo, profesorado, personal de administración y servicios, alumnado, madres y padres, así como también agentes exteriores al centro) pueden participar (expresarse, proponer, debatir, votar o deliberar) sobre el máximo de cuestiones que consideren pertinentes.

No rechazamos este nuevo sentido que se ha asignado al término "gobernanza", sino que la consideramos una concreción más (sumamente interesante, políticamente atractiva y por la que los miembros del equipo de investigación apostamos) de la idea genérica gobernanza que hemos definido. Para una mejor definición de los conceptos que proponemos y su fundamentación en los discursos en torno a la democracia elaborados desde la filosofía, la sociología y la politología véase Feu et al. (2013). 
b. Existen mecanismos para que todos los miembros de la comunidad educativa conozcan de antemano los cauces de participación. Se presta una especial atención al profesorado novel, así como al alumnado y familias en riesgo de exclusión social.

c. La mayoría de los miembros de la comunidad educativa participa de manera habitual en los espacios y procesos de toma de decisiones.

\subsubsection{Espectro de cuestiones susceptibles de ser acordadas democráticamente}

a. Todos los temas pueden ser debatidos por el conjunto de la comunidad educativa. No hay temas que se excluyan del debate o para los que el debate sea solamente posible entre los miembros de un determinado sector de la comunidad.

b. Existen criterios para establecer qué temas deben ser decididos por una parte de la comunidad.

c. Los criterios y normas de deliberación pueden ser revisados y sometidos a discusión entre los miembros de la comunidad educativa, con las limitaciones que establezca el marco legal (que se puede desarrollar e interpretar de modo que favorezca la máxima participación).

\subsubsection{Calidad de la participación}

a. Se evitan, en la medida de lo posible, las reuniones meramente informativas. Se apuesta al máximo por la amplia existencia de espacios deliberativos, de contraste de opiniones y de toma de decisiones.

b. Existen espacios y tiempos suficientes para que los distintos sectores de la comunidad educativa integren la praxis deliberativa como dinámica habitual.

c. Todas las personas convocadas a participar pueden intervenir en el establecimiento de los aspectos que se van a tratar (por ejemplo, se pueden proponer temas o participar en el establecimiento del orden del día).

d. Los miembros convocados disponen de la información sobre los aspectos que se someten a discusión con antelación suficiente, con el fin de asegurar una participación informada y un proceso de auténtica deliberación.

e. Los acuerdos tomados en los órganos y espacios de participación son debidamente registrados. Asimismo, se toman las medidas necesarias para que la información en general y los acuerdos adoptados lleguen a todos los miembros de la comunidad educativa, de tal manera que se puedan establecer dinámicas de rendición de cuentas y de control de los acuerdos y de su ejecución.

f. Se establecen mecanismos con el fin de asegurar que los acuerdos adoptados se apliquen efectivamente (asignación de responsabilidades, establecimiento de calendarios, etc.).

g. Se establecen dinámicas para fomentar el aprendizaje de las formas de participación democrática, dirigida tanto al alumnado como al resto de la comunidad educativa.

h. La mayoría de los miembros de la comunidad educativa expresa satisfacción ante los espacios de participación y los procesos de toma de decisiones existentes en el centro.

\subsection{HABITANZA}

\subsubsection{Definición}

La habitanza, tal como la definimos en Feu, Prieto y Simó (2016), se refiere a las condiciones y formas de vida de las personas, que definen su calidad de vida y que permiten - aunque no garantizan — una vida en libertad. 
Las condiciones de habitanza hacen posible que todos los miembros de la sociedad (en nuestro caso, el alumnado del centro educativo) tengan las mismas posibilidades de desarrollar sus capacidades y que la igualdad no sea solo una declaración formal y jurídica. Permiten que uno pueda liberarse de la permanente preocupación respecto a las necesidades básicas, y a su vez, atender el desarrollo personal (en nuestro caso, el estudio, la reflexión, el juego, las relaciones personales, etc. del alumnado) y también preocuparse por lo público (lo que hace que la gobernanza no sea una actividad exclusivamente para pudientes).

Consideramos formas de habitanza democrática tanto los principios de la educación inclusiva como la calidad de la acogida de los centros y todo aquello que contribuya a que la comunidad educativa, y muy especialmente los alumnos, puedan sentirse bien y estén en condiciones de cumplir con su principal cometido (ser ciudadanos autónomos y felices, con criterio, capaces de relacionarse adecuadamente con los demás y de superar con éxito los distintos tramos del sistema educativo).

Pero la habitanza no se refiere únicamente a las condiciones mínimas de calidad de vida del alumnado que podemos relacionar con ciertas ideas de justicia social (alimentación, vestido, vivienda, educación, seguridad, trabajo, acceso a la información, etc.). Se refiere también a aquellas condiciones que, de una forma amplia, tienen que ver con el estar en el mundo y con los otros: la hospitalidad, la cordialidad, la amistad, la armonía de los espacios y los tiempos, también son factores importantes en el desarrollo personal y social.

\subsubsection{Indicadores de una habitanza escolar democrática}

4.2.2.1. Las condiciones mínimas de calidad de vida del alumnado relacionadas con ciertas ideas de justicia social

a. El centro ha establecido mecanismos que le permiten detectar, y si es necesario atender (o, en su caso, derivar a servicios especializados), problemáticas de: alimentación, adquisición de libros de texto o material escolar, dificultades económicas para realizar actividades escolares o extraescolares, salud, vivienda, convivencia familiar, malos tratos o abusos.

Se trata de observar si el centro dispone y hace uso de mecanismos que le permitan intervenir ante situaciones en las que las necesidades básicas del alumnado no están cubiertas. Determinar, por ejemplo si las familias (o instituciones substitutorias) pueden atender las condiciones de vida básicas de sus hijos, los alumnos de los centros educativos. Son aspectos muy diversos, alguno de los cuales pueden ser considerados privados y externos a las responsabilidades educativas de los centros, pero que en tanto que afectan y condicionan directamente a su función educativa, no pueden considerarse ajenas. En este sentido, los centros escolares deben disponer y hacer uso de recursos instrumentales dirigidos a detectar o paliar este tipo de problemáticas que lesionan derechos fundamentales, que impiden o dificultan la función formativa y educativa de los centros y que minan la cohesión, la integración y el sentido de comunidad.

\subsubsection{La buena calidad de la acogida, la convivencia y el bienestar en el centro}

a. El centro dispone de protocolos o planes de actuación para acoger:

- a los alumnos, especialmente a los que se encuentran en situación de exclusión social o en riesgo de exclusión.

- a las familias, especialmente a las que se encuentran en situación de exclusión social o en 
riesgo de exclusión.

- al profesorado, especialmente al profesorado novel.

b. El centro dispone de medidas de atención y protección del personal laboral, garantiza sus derechos laborales y aporta cuantos recursos tenga al alcance para facilitar, en la mayor medida posible, la conciliación familiar y laboral.

c. El centro dispone de infraestructuras suficientes y adecuadas para llevar a cabo la tarea educativa:

- La arquitectura y decoración del centro educativo, en su conjunto, es agradable, acogedora e invita a estar en él.

- Los espacios del centro (aulas, salas de reuniones, patio, etc.) son suficientes, funcionales, cómodos, no están masificados y tienen una estética agradable.

- Se dispone de espacios suficientes y adecuados para la participación democrática y la toma de decisiones de toda la comunidad educativa.

- El centro es funcional y está preparado para su uso por parte de personas con todo tipo de discapacidades.

- El equipamiento y mobiliario son adecuados, están cuidados y son funcionales.

d. El centro dispone de recursos humanos suficientes para llevar a cabo el cometido educativo.

e. El centro dispone de recursos económicos suficientes para llevar a cabo el cometido educativo.

f. El centro cuenta con material pedagógico y didáctico adecuado y suficiente.

4.2.2.3. El trabajo orientado a que todo el alumnado adquiera las capacidades y conocimientos necesarios para desarrollar sus capacidades y vivir en sociedad

a. El centro dispone de recursos suficientes que le permiten atender adecuadamente a todos los alumnos y, especialmente, a los que puedan tener más dificultades para alcanzar los objetivos educativos establecidos para todos.

b. En el centro se han establecido los protocolos, se han adoptado formas de trabajo o se han distribuido los recursos (de profesorado, de espacio o del tipo que sea) con el fin de atender adecuadamente a todos los alumnos y, especialmente, a los que puedan tener más dificultades para alcanzar los objetivos educativos establecidos para todos.

Entendemos por éxito educativo o escolar que todo el alumnado adquiera los conocimientos y competencias obligatorios que permiten avanzar en el sistema educativo, así como disponer de la libertad y la capacidad crítica necesarias para el desarrollo personal y social. Que el alumnado tenga aseguradas unas condiciones materiales y afectivas básicas, que el centro disponga de las condiciones materiales y humanas necesarias y que goce de un buen clima, son condiciones imprescindibles, pero no suficientes para el éxito educativo. Es necesaria también una buena acción educativa.

\subsection{ALTERIDAD}

\subsubsection{Definición}

La alteridad se refiere a las acciones, políticas y programas que hacen posible el reconocimiento normalizado, digno y positivo de personas y colectivos no hegemónicos y, que por esta condición, fácilmente quedan invisibilizados, estigmatizados o representados de forma negativa. Este reconocimiento puede ser emocional (fomentando relaciones de amor y amistad, que posibilitan la autoconfianza), jurídico (estableciendo y dando 
base legal a los principios de igualdad y de respeto a la diversidad, que posibilitan un autorrespeto elemental) y social (potenciando el establecimiento de relaciones sociales a partir de la consideración y valoración hacia los demás, que posibilitan la autoestima). ${ }^{7}$

La democracia, desde la dimensión de la alteridad, nos enfrenta al difícil equilibrio entre el principio de igualdad y el respeto a la diversidad; así como al equilibrio, también difícil, entre los derechos individuales y el derecho al reconocimiento de determinados colectivos. ${ }^{8}$ Desde esta perspectiva, no basta con garantizar la igualdad entre las personas cuando estas pertenecen a colectivos con diferentes recursos, prestigio y reconocimiento social. Asimismo, se considera que debe velarse para que no se imponga la igualdad a todos, de manera arbitraria, aboliendo formas de diversidad (lingüística, sexual, cultural o del tipo que sea), sino que estas deberían ser reconocidas y respetadas siempre que no vulneren derechos fundamentales. Por consiguiente, la inclusión, entendida como un derecho humano (Parrilla, 2002), lucha por la participación en igualdad de condiciones en todas las instituciones sociales, considerándola un hecho de justicia social y un derecho inalienable en las sociedades democráticas (Ballard y McDonald, 1998; Clough y Corbett, 2000; Grossman, 2008).

\subsubsection{Indicadores de una alteridad escolar democrática}

La mayoría de las propuestas de la "educación intercultural" (o "multiculturalismo crítico", en la tradición británica) y las prácticas de acogida y atención a la diversidad desde una perspectiva inclusiva, se pueden considerar como iniciativas que permiten trabajar la democracia desde la perspectiva de la alteridad. Con el fin de centrarnos en la alteridad como dimensión de la democracia en los centros educativos, proponemos que se concrete en las prácticas, discursos, iniciativas, políticas o proyectos que se establecen con el fin de dar visibilidad, reconocer, respetar, valorar positivamente, acoger e incluir al "otro" (el otro minoritario, el otro no convencional, el otro contrahegemónico, etc.).

Ante la dificultad de delimitar dichos conceptos, proponemos que se indague en el desarrollo de tales "prácticas de reconocimiento" (por decirlo de un modo abreviado) en los distintos ámbitos que se señalan a continuación:

\subsubsection{La consideración de la alteridad ante los procesos de representación política}

a. Las personas que representan intereses no hegemónicos (especialmente las más próximas a sufrir situaciones de marginación o exclusión social), están presentes, representadas y se tienen en cuenta en igualdad de condiciones, en todos los espacios de participación (institucionalizados y no institucionalizados).

En este sentido, seguimos a Axel Honneth (1997) quien propone que reconozcamos esta triple dimensión del reconocimiento: jurídico (que según él, posibilita el autorrespeto), social (que posibilita la autoestima) y emocional (que hace posible la autoconfianza).

8 Como señalamos en Feu et al. (2013), esta colisión aparente de sujetos de derecho ha sido puesta de relieve por algunos autores liberales (los ataques de Sartori (2001) al multiculturalismo han sido unos de los que más impacto han tenido tanto en el ámbito de la política como entre el público en general), si bien también desde el liberalismo se ha hecho una sólida defensa de la alteridad, entendida como apuesta por el reconocimiento y el respeto a las identidades y los derechos de determinados colectivos (al frente de estos autores de tradición liberal podemos situar al canadiense Will Kymlicka (1995a, b)). 


\subsubsection{La consideración de la alteridad ante los procesos de participación social}

a. El centro dispone de un plan, propuesta o protocolo para favorecer la asistencia de familias diferentes a los acontecimientos comunitarios y festivos abiertos a toda la comunidad educativa (fiestas, jornadas, encuentros, etc.) y para que, independientemente de la realidad del centro, no haya actos ni se produzcan discursos que atenten contra la integridad y se respeten las situaciones de familias con dificultades o en riesgo de exclusión.

\subsubsection{Visualización de la diversidad en los centros}

a. La diversidad de la comunidad educativa se refleja en los órganos de participación y de toma de decisiones.

b. La diversidad de la comunidad educativa se refleja en la vida cotidiana del centro (decoración, actos públicos, etc.).

4.3.2.4. Adaptaciones organizativas que promueven el desarrollo de una educación inclusiva

a. El centro planifica y desarrolla acciones educativas para reducir situaciones de discriminación o exclusión entre el alumnado.

b. Las minorías culturales o religiosas con especificidades alimentarias son atendidas con toda normalidad — como lo es el colectivo mayoritario — en el comedor escolar, y de ninguna manera son tratadas como grupos especiales.

c. Se reducen las barreras de accesibilidad para todos los miembros de la comunidad educativa en todos los espacios del centro.

4.3.2.5. Adaptaciones académicas (currículum, dinámicas de aula o prácticas de enseñanza y aprendizaje) que promueven el desarrollo de una educación inclusiva

a. El centro dispone de un currículo que visibiliza positivamente y denuncia el silencio histórico —estigma o prejuicio- de las personas y grupos con atributos y reivindicaciones no hegemónicos, y hace cuanto está a su alcance para que estas personas y grupos dejen de ser percibidos según dicho estigma o prejuicio.

b. La visualización positiva y, en la medida de lo posible, la denuncia del silencio histórico respecto a las personas y grupos con atributos o reivindicaciones no hegemónicos no solo se refleja en la concreción curricular, sino también en el material escolar y didáctico — libros de texto, juegos, material bibliográfico, material informativo- presente en la clase o escuela.

c. El centro promueve metodologías de enseñanza-aprendizaje en las que la heterogeneidad se asume como un valor.

\subsection{VALORES, VIRTUDES Y CAPACIDADES}

\subsubsection{Definición}

Barbosa (2000) y Edelstein (2011) consideran que las aportaciones sobre los valores y la educación democrática están íntimamente relacionadas con lo que podríamos denominar "educación cívica", "civismo" o "la educación para la ciudadanía”. Para que la gobernanza, la habitanza y la alteridad funcionen según parámetros democráticos, son necesarios unos 
valores, virtudes y caracteres determinados. Así pues, el éthos (en buena medida aquello de lo que viene ocupándose desde hace siglos la educación) es parte constitutiva y fundamental de las otras tres dimensiones a las que nos hemos referido. Así se asume desde la tradición republicana: no puede haber deliberación genuina (ni participación valiosa ni deseable) sin ciudadanos conscientes de sus deberes y responsabilidades, competentes, activos, bien informados y dispuestos a comprometerse y actuar al servicio del bien público (Peña, 2000) $)^{9}$. Desde esta perspectiva, el éthos democrático comprende el conjunto de valores, virtudes y capacidades que se deben promover entre los ciudadanos para que estos no sean objetos pasivos de la política y puedan transformarse en ciudadanos activos, capaces de participar en los procesos democráticos y defenderlos tanto frente a los que se oponen a ellos, como frente a aquellos que pervierten su funcionamiento o lo alejan de su principal cometido, que no es otro que servir al bien común (Skinner, 2004).

\subsubsection{Indicadores de un éthos escolar democrático}

Se trata de valores, virtudes y capacidades que habilitan a los ciudadanos para hacer un buen uso de los mecanismos de gobernanza democrática (respeto de la libertad y las opiniones de los demás; capacidad de escuchar, de expresarse, de interpretar y contrastar la información; espíritu crítico) indispensables para la defensa de los principios de habitanza democrática (responsabilidad, compromiso, prudencia, defensa de la justicia y el bien común, capacidad de resolver los conflictos de manera pacífica) o los capacitan para el reconocimiento de la alteridad (tolerancia, solidaridad, respeto a las minorías, capacidad de diálogo y acomodo en sociedades diversas), entre otros.

Considerando las características establecidas para el desarrollo de la educación cívica, proponemos agrupar los indicadores de un éthos escolar democrático según los apartados siguientes:

a. Se ha debatido y existe un acuerdo general entre los miembros de la comunidad escolar sobre los valores que se van a promover y que van a regir la vida de los centros.

b. Los valores, virtudes y capacidades democráticas se promueven a través del currículum explícito y oculto.

c. Los valores, virtudes y capacidades democráticas se promueven a través de la vivencia e implementación de prácticas democráticas en el centro, vinculadas a procesos organizativos o de enseñanza y aprendizaje.

\section{CONCLUSIONES Y REFLEXIONES PARA EL DEBATE}

El término democracia es un concepto polisémico y controvertido. Esta primera constatación debe entenderse, más que como un problema o una limitación, como una riqueza y una oportunidad: nos da la posibilidad de practicarla de distintas maneras, por lo que hace honor a los valores de pluralidad, respeto, participación y creatividad a que va asociada. El problema aparece cuando se establece una breve definición totalizadora

Léase, por ejemplo, a Agulló (2014), Ovejero, Martí y Gargarella (2004), Pettit (1998), Sandel (1996) o Skinner (2004). También algunos autores inscritos en la tradición de pensamiento liberal, como Macedo (1990), Galston (1991) o Rawls (1993) han aportado algunas reflexiones en torno a la función de las virtudes cívicas desde principios liberales. 
de un concepto tan complejo, y se considera que debe ser seguido por toda la comunidad educativa. Si se nos preguntase qué es la escuela democrática, responderíamos que es la imagen resultante y abierta de la superposición, a modo de transparencias, de todas las dimensiones e indicadores que se han presentado en este artículo y mucho más.

La segunda cuestión a tener en cuenta es que, a tenor de las conclusiones establecidas en la introducción de este artículo (la situación de la democracia en los centros educativos españoles), se transmita una visión pesimista. Es cierto que el estado general de la democracia en los centros educativos es más bien pobre, de baja intensidad. Ahora bien, esto no impide que haya experiencias — seguramente pocas, o menos de las que desearíamosque superan la realidad hegemónica. En toda la geografía española hay centros públicos, concertados y privados que hacen cuanto está a su alcance y más para promover prácticas democráticas. Algunos se limitan a reformar los órganos de participación y toma de decisiones, otros crean órganos ad hoc para potenciar una participación que no es posible en los órganos estandarizados, y otros van mucho más allá a través de proyectos educativos integrales. ${ }^{10}$

Las dinámicas institucionales actuales poco favorecen lo que convenimos en llamar la "otra" democracia. El discurso de la Administración, por una parte, y las prácticas hegemónicas, por otra, han contribuido y contribuyen a socavar - a veces de forma sutil y otras de manera desacomplejada - la riqueza, la controversia y la profundidad de lo que aquí nos ocupa. Cuando en la gramática docente se instala la obsesión de "rendir cuentas", de los "rankings", de la financiación externa, de la competición; cuando se introduce el gerencialismo en los centros educativos, cuando estos se equiparan a una organización empresarial y se rigen por principios de economía liberal, cuando alumnos y familias pasan a ser clientes, es difícil hablar de democracia en profundidad.

Con demasiada frecuencia nos quejamos de que falta una concepción teórica y precisa de lo que entendemos por democracia, y de la improvisación que se da en los centros a la hora de diseñar procesos democráticos que superen las prácticas dominantes. En estas páginas hemos realizado una propuesta, compleja (como el mismo concepto de democracia), pero operativa y realista. Hemos considerado que no puede hablarse de plena democracia en los centros y en la comunidad educativa si no se atienden y desarrollan cuatro dimensiones fundamentales: la gobernanza, la habitanza, la alteridad y el éthos. Y de cada una de ellas se han señalado aspectos que las concretan y los indicadores para valorar la calidad democrática de un centro.

La democracia en la escuela es, sin duda, un aspecto que un país que se define como democrático no puede eludir, ya que conlleva la supervivencia democrática. La administración educativa debería poner todos los medios necesarios, recursos e imaginación, para que sea una realidad significativa en la comunidad educativa. Ningún colectivo (profesionales de la enseñanza, alumnado, familias, personal de administración y servicios, etc.) queda excluido de esta responsabilidad.

Para que un centro educativo profundice en la democracia, sugerimos un proceso de permanente reflexión colectiva. Probablemente, el primer paso sea hacer explícito este interés por parte de quienes tienen la responsabilidad del centro (equipo directivo

Valga como sugerencia la consulta de la página web http://governacio.gencat.cat/ca/pgov_ambits_d_actuacio/qualitat_ democratica_i_participacio/enfortiment-societat-civil/premi_participa_a_1_escola en la que se encuentran, entre otras cosas, escuelas de primaria premiadas por las acciones de mejora democrática que han realizado. 
y claustro). Esta manifestación posibilita el debate teórico — a poder ser compartido por todos y todas - de aspectos sustantivos que orienten el tipo de democracia por el que se apuesta. La discusión teórica puede y debe ser, entre otras cosas, abierta y plural, ya que las distintas orientaciones que puedan surgir responden a proyectos políticos y sociales diferenciados. Al final, sin embargo, será necesario hallar un marco de encuentro en el que la comunidad educativa se sienta cómoda y lo acepte. En cualquier caso, recomendamos construir un discurso teórico multidimensional que transcienda la estricta dimensión de la gobernanza para ahondar en las apasionantes dimensiones de la habitanza, la alteridad y el éthos que aquí se han presentado. A raíz del momento social, político y económico que estamos viviendo a escala planetaria, es importante que el debate teórico que proponemos incorpore elementos que, a priori, parece que no forman parte de los aspectos nucleares de la democracia, pero que, como hemos argumentado, no cabe duda de que son parte de ella.

Las conclusiones derivadas de dicho debate teórico deben fundamentar el modelo de centro democrático y comprometido (no vale apostar por la falsa neutralidad), y con una opción clara por lo común y la comunidad, sin olvidar la imprescindible individualidad. Recomendamos concluir con un relato ambicioso y elevado, sin olvidarse de que dicho relato debe acompañarse de una voluntad práctica que materialice el proyecto democrático dándole visibilidad, practicidad y objetividad.

La urgencia de replantearnos la democracia en los centros educativos viene apremiada por factores que con demasiada frecuencia son olvidados: las nuevas formas de participación de los jóvenes y la reivindicación de una democracia más participativa que, en nuestro país, arranca con fuerza a partir del movimiento 15M (gobernanza); el incremento de las desigualdades sociales y, por lo tanto, de las situaciones de injusticia de las familias más vulnerables económicamente y socialmente (habitanza); el aumento de la pluralidad y diversidad cultural de nuestras sociedades (alteridad); y la necesidad y riqueza de vivir juntos, conservando la propia identidad sin considerar extraño al diferente (éthos). Compartimos el análisis de Candia y Gomes (2013, p. 53) cuando afirman que: "El sentido de ciudadanía que coincide con el punto de vista democrático refuerza los encaminamientos de las políticas educativas para que su efectividad dé sentido a la constitución de ciudadanos que vivan y reclamen el espacio social como conquista universalizada y humanizada, objeto que es el mayor desafío de las sociedades contemporáneas, debido al predominio de la disposición estructural del capitalismo". El camino tomado nos da la oportunidad de transformar las dificultades en posibilidades para seguir avanzando hacia una escuela más democrática.

\section{REFERENCIAS BIBLIOGRÁFICAS}

Agulló, M.V. (2014). La democracia republicana: problemas y límites de un modelo alternativo a la democracia liberal. REIS: Revista Española de Investigaciones Sociológicas, 146, 217-238.

Apple, M. W. (2011). Democratic education in neoliberal and neoconservative times. International Studies in Sociology of Education, 21(1), 21-31.

Apple, M. W., \& Beane, J. A. (1999). Escuelas democráticas. Madrid: Morata.

Apple, M. W., \& Beane, J. A. (2007). Democratic schools: Lessons in powerful education. Portsmouth, NH: Heinemann.

Ballard, K., \& MacDonald, T. (1998). New Zealand: Inclusive school, inclusive philosophy?. En T. Booth, \& M. Ainscow (Eds.), From them to us: an international study of inclusion in education (pp. 68-94). London: Routledge. 
Barber, B. (2004). Democracia fuerte. Política participativa para una nueva época. Córdoba: Almuzara.

Barbosa, M. (2000). Educar per a una ciutadania democràtica a les escoles: una discussió de models. Temps d'Educació, 24, segundo semestre, 359-373.

Biesta, G. (2009). Sporadic Democracy: Education, Democracy and the Question of Inclusion (Chapter 7). In M. S. Katz, S. Verducci \& G. Biesta (Eds.), Education, Democracy, and the Moral Life (pp. 101-112). Netherlands: Springer. doi:10.1007/978-1-4020-8626-7.

Bobbio, N., Matteuci, N., \& Pasquino, G. (1991). Diccionario de política. Madrid: Siglo XXI.

Brown, W. (2010). Ahora todos somos demócratas. En G. Agamben, A. Badiou, D. Bensaïd, W. Brown, J. L. Nancy, J. Rancière, K. Ross \& S. Zizek (Eds.), Democracia en suspenso (pp. 5978). Madrid: Casus Belli.

Candia, L.T., \& Gomes, P. (2013). Ciudadanía, educación y la realidad brasileña: Puntos de debate y encaminamiento. Estudios Pedagógicos, XXXIX(2), 41-54.

Clough, P., \& Corbett, J. (Ed.). (2000). Theories of inclusive education. Londres: Paul Chapman.

De Francisco, A. (1998). Republicanismo y modernidad. Claves de Razón Práctica, 95, 42-48.

Dewey, J. (1895). Plan of organization of the university primary school. In Early works of John Dewey (Vol. 5, pp. 224-243). Carbondale: Southern Illinois University Press.

Edelstein, W. (2011). Education for Democracy: reasons and strategies. European Journal of Education, 46(1), 127-137. doi:10.1111/j.1465-3435.2010.01463.x

Elster, J. (Comp.). (2001). Democracia deliberativa. Barcelona: Gedisa.

Feito, R. (2010). Democracia participativa frente a segregación y racismo en una época de crisis económica. Revista de la Asociación de Sociología de la Educación, 3(1), 20-40.

Feito, R. (2014). Treinta años de Consejos Escolares. La participación de los padres y de las madres en el control y gestión de los centros sostenidos con fondos públicos. Profesorado. Revista de currículum y formación del profesorado, 18(2), 51-67.

Feito, R., \& López Ruiz, J. I. (2008). Construyendo escuelas democráticas. Barcelona: Hipatia.

Fernández Enguita, M. (1992). Poder y participación en el sistema educativo. Barcelona: Paideia.

Feu, J., Prieto, O., \& Simó, N. (2016). ¿Qué es una escuela verdaderamente democrática? Cuadernos de Pedagogía, 465, 90-97.

Feu, J., Serra, C., Canimas, J., \& Simó, N. (2013). El concepto teórico de democracia aplicado a la educación: modelos teóricos y consecuencias de los mismos. Comunicación presentada al XI Congreso Español de Sociología 2013. Madrid. Recuperado de http://www.fes-web.org/ congresos/11/ponencia/1496/

Fishkin, J. (1995). Democracia y deliberación. Nuevas perspectivas para la reforma democrática. Barcelona: Ariel.

Fishkin, J. (2009). When the People Speak. Deliberative Democracy and Public Consultation. Nueva York: Oxford University Press.

Galston, W.A. (1991). Liberal purposes: goods, virtues, and diversity in the liberal state. Cambridge; New York: Cambridge University.

Garreta, J. (2009). La participación de las familias en la escuela pública. Las asociaciones de madres y padres del alumnado. Madrid: CEAPA.

Gil Villa, F. (1995). La participación democrática en los centros de enseñanza no universitarios. Madrid: MEC.

González, J. F. (2004). Los consejos escolares de los colegios de educación primaria analizados por sus miembros. Educación XXI. Revista de la Facultad de Educación, 7, 87-110.

Grossman, D. L. (2008). Democracy, citizenship education and inclusion: a multidimensional approach. Prospects, 38, 35-46.

Habermas, J. (1999). Tres modelos normativos de democracia. En La inclusión del otro. Estudios de teoría política (pp. 231-246). Barcelona: Paidós.

Honneth, A. (1997). La lucha por el reconocimiento. Por una gramática moral de los conflictos 
sociales. Barcelona: Crítica.

Kesby, M., Kindon, S., \& Pain, R. (2007). Participation as a Form of Power: Retheorising Empowerment and Spatialising Participatory Action Research. En S. Kindon, R. Pain., and M. Kesby (Eds.), Participatory Action Research Approches and Methods: Connecting People, Participation and Place (pp. 19-25). London: Routledge.

Kymlicka, W. (1995a). Multicultural Citizenship. Oxford: Claredon Press.

Kymlicka, W. (1995b). The Rights of Minority Cultures. London: Oxford University Press.

Leithwood, K. A., Begley, P., \& Cousins, B. (1990). The nature, causes and consequences of principals' practices: an agenda for future research. Journal of Educational Administration, 28(4), 5-31.

Macedo, S. (1990). Liberal Virtues: Citizenship, Virtue and Community in liberal Constitutionalism. Oxford: Claremont Press.

Martí, J. L. (2006). La república deliberativa. Una teoría de la democracia. Madrid: Marcial Pons.

Martínez Bonafé, A., Molina, D., \& Montaner, C. (2003). Vivir la democracia en la escuela. Una manera de formular los problemas del aula y del centro. Tabanque, 17, Valladolid: Universidad de Valladolid.

Ministerio de Educación, Cultura y Deporte. Consejo Escolar del Estado. (2013). Informe 2013 sobre el estado del sistema educativo. Curso 2011-2012, 602-604. Madrid: Secretaría General Técnica. Subdirección General de Documentación.

Ovejero, F., Martí, J.L., \& Gargarella, R. (Comps.). (2004). Nuevas ideas republicanas. Autogobierno y libertad. Barcelona: Paidós Estado y Sociedad.

Parrilla, A. (2002). Acerca del origen y sentido de la educación inclusiva. Revista de Educación, 327, 11-29.

Peña, J. (2000). La ciudadanía hoy. Problemas y propuestas. Valladolid: Universidad de Valladolid.

Pettit, P. (1998). Republicanismo. Una teoría sobre la libertad y el gobierno. Barcelona: Paidós.

Rawls, J. (1993). Political Liberalism. New York: Columbia University Press.

Rhodes, R.A.W. (1997). Understanding Governance. Buckingham: Open University press.

Sandel, M. (1996). Democracy's Discontent: America in Search of a Public Philosophy. Cambridge: Cambridge University Press.

Sandel, M. (1998). Reply to critics. In A. Allen, \& M. Regan (Eds.), Debating Democracy's Discontent: Essays on American Politics, Law and Public Philosophy. New York: Oxford University Press.

Santos Guerra, M. A. (1999). El crisol de la participación. Estudio Etnográfico sobre la participación en Consejos Escolares de Centro. Málaga: Aljibe.

Sartori, G. (2001). La sociedad multiétnica: pluralismo, multiculturalismo y extranjeros. Madrid: Taurus.

Skinner, Q. (2004). Las paradojas de la libertad política. En R. Gargarella, J.L. Martí, \& F. Ovejero (Comps.), Nuevas ideas republicanas. Autogobierno y libertad. Barcelona: Paidós.

Subirats, J. (2010). Si la respuesta es gobernanza, ¿Cuál es la pregunta? Factores de cambio en la política y en las políticas. Ekonomiaz, 74, 16-35.

Subirats, J. (2011). Otra sociedad ¿Otra política? De "no nos representan” a la democracia de lo común. Barcelona: Icaria.

Taylor, C. (1979). What's Wrong with Negative Liberty? In A. Ryan (Comp.), The Idea of Freedom. Oxford: Oxford University Press.

Viroli, M. (2002). Republicanism. New York: Hill \& Wang. 
